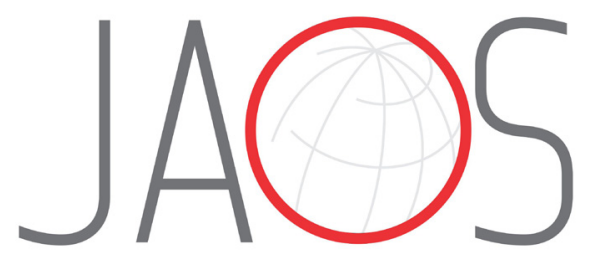

\title{
Chlorhexidine for prevention of alveolar osteitis: a randomised clinical trial
}

\section{Abstract}

Diego HALABI ${ }^{1}$

Jose ESCOBAR ${ }^{2}$

Cyntia ALVARADO 1

Nicolette MARTINEZ ${ }^{1}$

Carlos MUÑOZ1
Submitted: June 21, 2017 Modification: September 8, 2017

Accept: September 27, 2017

Corresponding address: Diego Halabi

Universidad Austral de Chile, Facultad de Medicina, Escuela de Odontologia, Valdivia, Chile. Phone: +56 632293928 e-mail: diego.halabi@uach.cl
Objective: To determine the effectiveness of chlorhexidine $0.12 \%$ mouthwash (CHX) after tooth extraction for the prevention of alveolar osteitis (AO). Material and methods: We conducted a double-blind randomised clinical trial stratified by risk factors. We enrolled a cohort of 822 patients who underwent dental extractions, and were considered to be at risk of developing $A O$ (previous surgical site infection, traumatic extraction, and tobacco smoking). After extraction, patients were randomly allocated for $\mathrm{CHX}$ group or placebo group, matched by risk factors. The primary outcome was clinical diagnosis of $\mathrm{AO}$ : increasing postoperative pain for $4 \mathrm{~d}$ within and around the socket, and total or partial breakdown of the blood clot in the socket with or without bone exposure. Results: Follow-up was completed by 744 participants ( 372 chlorhexidine and 372 placebo). We detected no significant differences between the two groups at baseline. After completed follow-up, risk factors were equally distributed between the two groups. Overall incidence of OA was $4.97 \%$, in which 27 participants treated with placebo $(7.26 \%)$ and 10 participants treated with $\mathrm{CHX}(2.69 \%)$ developed AO. $\mathrm{CHX}$ reduced the incidence of $\mathrm{AO}$ by $63 \%$ [Absolute Risk Reduction: 4.57 (95\% CI 1.5-7.7), Number Needed to Treat: 21.88 (95\% CI 13.0-69.3), Fisher's exact test: $p=0.006]$. No adverse effects were reported. Conclusion: The use of chlorhexidine $0.12 \%$ mouthwash after tooth extraction is safe and effective in reducing the incidence of $A O$ in high-risk patients.

Keywords: Preventive dentistry. Oral surgery. Tooth extraction. Chlorhexidine. Clinical trial. Alveolar osteitis. 


\section{Introduction}

Patients who undergo dental extractions are at risk of developing post-operative complications, and the most common is the alveolar osteitis $(A O)^{12}$. Our group ${ }^{8}$ recently reported an incidence of $6.4 \%$ of $A O$, and we determined by a logistic regression model that previous surgical site infection, tobacco smoking and traumatic extraction are risk factors for developing $A O$. These risk factors explain why there were changes in prevalence ranging from $3.9 \%$ up to $29.6 \%$ for the third molar ${ }^{10,23}$.

With a risk model that predicts the development of $A O$, it is possible to implement preventive health care to those individuals at high risk. Thus, clinicians can help their patients to avoid the severe pain caused by this complication ${ }^{17}$, which traditionally receives symptomatic treatment of uncertain effectiveness ${ }^{16}$. Hence, clinicians can reduce health care costs and provide comfort for patients ${ }^{9}$.

It has been proposed that chlorhexidine $0.12 \%$ mouthwash can be used after extraction for the prevention of $\mathrm{AO}^{10,13,18}$. Chlorhexidine is the most widely used antiseptic in dentistry because its broad-spectrum antibacterial effectiveness is well established $^{2}$, so it can be implemented as a simple and inexpensively public health policy.

However, the clinical trials that support chlorhexidine $0.12 \%$ mouthwash are inconclusive, showing methodological weaknesses and having a high risk of bias $^{5,9,24}$. Therefore, the need to conduct randomized clinical trials of better quality and including risk factors is imperative.

Here, we conducted a double-blind randomised clinical trial stratified by risk factors to determine the effectiveness of $\mathrm{CHX}$ after tooth extraction for the prevention of $\mathrm{AO}$.

\section{Material and methods}

\section{Trial design}

We conducted a randomized, double-blind, parallelgroup, stratified by risk factors, placebo-controlled, clinical trial in two public community dental clinics in Valdivia, Chile (population 154,559). All participants agreed to participate by signing an informed consent form, according to the recommendations of the Declaration of Helsinki. The protocol of this study was approved by the Research Ethics Committee of the Public Health Service of Valdivia. The trial was registered as ISRCTN14646628.

\section{Population}

We recruited patients registered to receive dental care from a list of random numbers generated by computer, from April 2013 to December 2015. Inclusion criteria were adults of 18 years or older with clinical indications for tooth extraction, and who presented at least one of the following risk factors for developing $\mathrm{AO}$ : tobacco smoker (consumption of $\geq 5$ cigarettes $24 \mathrm{~h}$ before extraction), previous surgical site infection (clinical diagnosis of chronic periodontitis, acute periodontal conditions, apical periodontitis, pericoronitis, fungal infections, or dental pulp gangrene) and/or traumatic extraction (lifting a flap, use of elevators for $>4 \mathrm{~min}$, and/or rotary instruments).

Exclusion criteria were patients requiring extraction in the operating theater, residents of rural areas who manifested difficulty in returning for follow-up, patients allergic to chlorhexidine, patients under antimicrobial therapy, antibiotic prophylaxis, or antibiotics therapy after extraction.

Dental extractions were performed by dental surgery team from the emergency department of the clinic, in accordance with standard procedures as defined by the National Health Service ${ }^{14}$.

\section{Interventions}

After surgery, patients were allocated to the treatment group or the placebo group. Treatment consisted of a mouthwash with $15 \mathrm{ml}$ chlorhexidine $0.12 \%$ (Oralgene ${ }^{\circledR}$ Mouthwash $0.12 \%$, Maver, Chile) for $30 \mathrm{~s}$, twice a day for $7 \mathrm{~d}$, starting $24 \mathrm{~h}$ after extraction. The placebo was sterile water, with the same indications for use. Both chlorhexidine and placebo were stored in similar brown plastic bottles, and instructions were given orally and in writing to each participant.

To guarantee that in both groups (treatment and placebo) the risk of alveolar osteitis was similar and comparable, the assignment was performed by randomization, stratified by risk factors, and 7 groups were formed with the following possible combinations: smoker; previous infection; traumatic extraction; smoker + previous infection; smoker + traumatic extraction; previous infection + traumatic extraction; smoker + previous infection + traumatic extraction. 
To avoid the risk of having more patients in a group, we stored black envelopes in a box containing a paper with the letter $\mathrm{C}$ for chlorhexidine or $\mathrm{P}$ for placebo (half of each). The envelopes were chosen for each patient after the extraction and transported to another room (without opening them); they were read only by one of the authors, who then distributed the chlorhexidine or placebo accordingly. For each patient who was assigned to a group, the subsequent patient who arrived with the same risk factors was matched to the opposite group, and the respective envelope was discarded (to ensure homogeneity of groups).

\section{Outcome measures}

We recorded age (years), gender (male or female), tooth location (mandibular or maxillary), diagnosis or previous surgical site infection (yes or no, as described), smoking (smoker or non-smoker, as described) and traumatic extraction (yes or no, as described) before tooth extraction for each patient.

The primary outcome was positive diagnosis of $\mathrm{AO}$ one week after tooth extraction. Positive diagnosis of $A O$ was identified by the authors in patients with the following characteristics: 1) increasing postoperative pain intensity for $4 \mathrm{~d}$ within and around the socket and 2) total or partial breakdown of the blood clot in the socket with or without bone exposure.

At the same time, we assessed hypersensitivity to chlorhexidine (contact dermatitis, pruritus, vesicle formation, urticaria, dyspnea, or anaphylactic shock), dysgeusia (alteration of taste perception, bitter taste or burning) or pigmentation (staining of teeth and/or tongue) as potentially adverse events.

We treated patients who developed alveolar osteitis and other complications in accordance with the available clinical protocols of the Chilean Health Ministry ${ }^{14}$.

\section{Sample size}

We estimated sample size using data published previously by Halabi, et al. ${ }^{8}$ (2012), expecting an incidence reduction of two-thirds. The $P_{A}$ expected incidence of disease $(A O)$ in the placebo group was $6.14 \%$, while the $P_{B}$ expected incidence of disease (AO) in the $\mathrm{CHX}$ group was $2.05 \%$. Additionally, $k$ groups ratio of sample sizes between groups was $1: 1$. The power of the study was set at $80 \%(\beta=0.20)$, with $a=0.05$ as the significance level. Based on these parameters, we applied the following equation ${ }^{19}$ : sample size $=\left(\frac{p_{A}\left(1-p_{A}\right)}{k}+p_{B}\left(1-p_{B}\right)\right)\left(\frac{z_{1}-\frac{\alpha}{2}+z_{1}-\beta}{p_{A}-P_{B}}\right)^{2}$

\section{Statistics}

We performed the statistical analysis by $\mathrm{DH}$ using R 3.3.1 ( $R$ Foundation for Statistical Computing, Vienna, Austria). We used the Fisher's exact test to detect significant differences in the incidence of $A O$ between $\mathrm{CHX}$ and placebo groups, and also to analyse gender, tooth location, patient smoking, previous infection and traumatic extraction variables. We used unpaired t-test was to detect significant differences in age between $\mathrm{CHX}$ and placebo groups. We determined the incidence of $\mathrm{AO}$ for both groups and calculated the Number Needed to Treat (NNT). For all tests, statistical differences were determined to be significant at $\mathrm{p}<0.05$.

\section{Results}

From April 2013 to December 2015, we recruited 822 participants. Out of these, 744 met inclusion criteria and completed the follow-up. They were allocated into two groups of 372 for treatment of chlorhexidine $0.12 \%$ or placebo. Figure 1 shows the flow diagram of participants.

As seen in Table 1, we included 381 female participants and 363 males at baseline. The mean age was 43.43 years (SD 14.99). Comparison of baseline data between the group treated with chlorhexidine $0.12 \%$ and the one treated with placebo did not show statistically significant differences for age [ $t$ (degrees of freedom $)=0.917(742), p=0.359]$, gender $(p=0.463)$, location of the extracted tooth $(p=0.238)$, previous infection in surgical site $(p=0.999)$, tobacco smoke $(p=0.999)$ or traumatic extraction $(p=0.999)$.

Once all participants completed the follow-up, we observed that the risk factors were equally distributed between the two groups, without statistically significant differences (see details in Table 2).

We diagnosed 37 cases of $\mathrm{AO}$, with an overall prevalence of $4.97 \%$. In the group treated with chlorhexidine $0.12 \%$ mouthwash we diagnosed 10 participants $(2.69 \%)$ with $\mathrm{AO}$, while in the placebo group they were $27(7.26 \%)(p=0.006$, statistical power $=0.821)$. $\mathrm{CHX}$ reduced the incidence of $\mathrm{AO}$ by 63\% [Absolute Risk Reduction: 4.57 (CI95\% 1.5-7.7), Number Needed to Treat: 21.88 (CI95\% 13.0-69.3)]. 


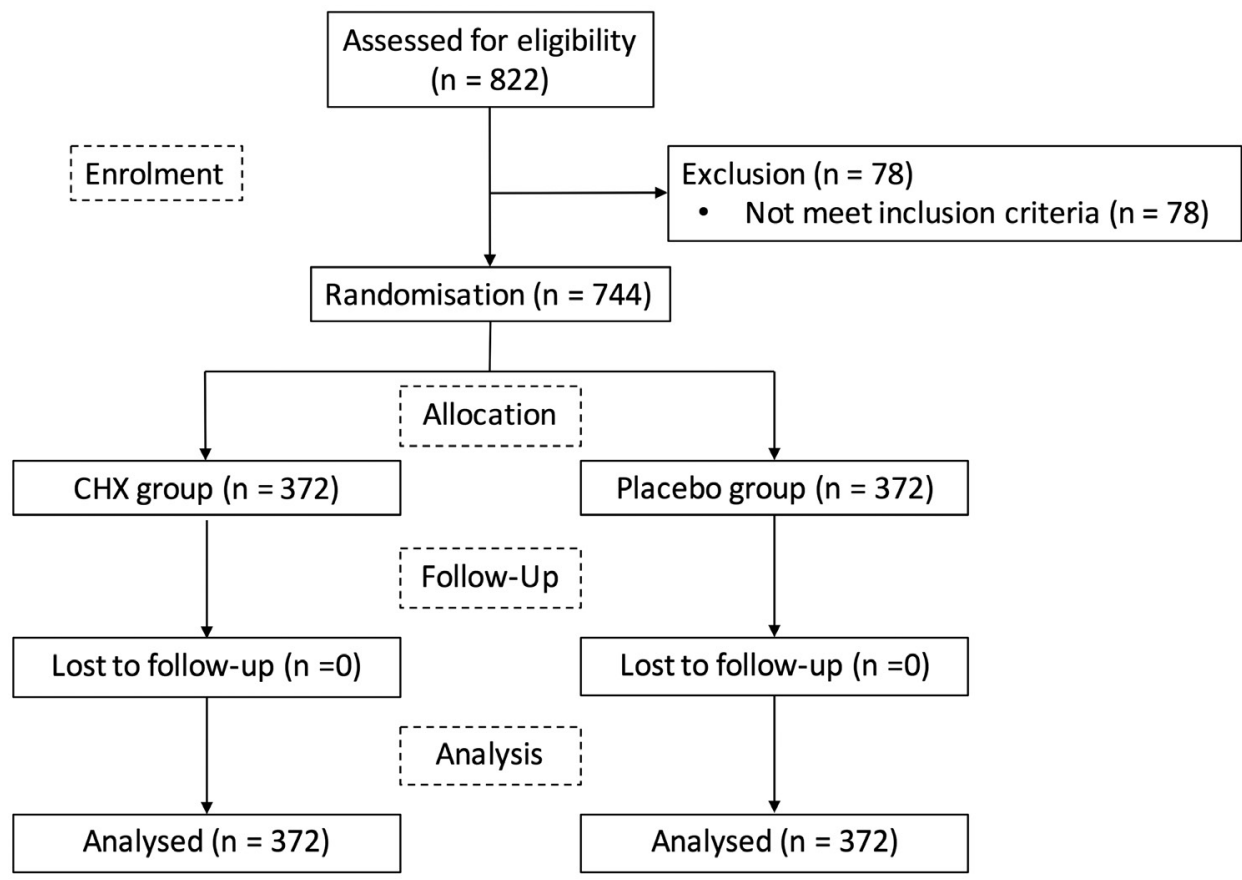

Figure 1- CONSORT flow diagram of patients included in the final analysis

Table 1- Baseline data of participants, mean age (SD), and frequency (\%) of gender, location of the tooth extracted, previous surgical site infection, tobacco smoke and traumatic extraction

\begin{tabular}{|c|c|c|c|c|}
\hline & $\begin{array}{c}\text { Chlorhexidine } \\
(n=372)\end{array}$ & $\begin{array}{l}\text { Placebo } \\
(n=372)\end{array}$ & $\begin{array}{c}\text { Total } \\
(n=744)\end{array}$ & $P$ value \\
\hline Age (years $\pm S D$ ) & $43.93 \pm 15.15$ & $42.92 \pm 14.84$ & $43.43 \pm 14.99$ & 0.396 \\
\hline \multicolumn{5}{|l|}{ Gender [n (\%)] } \\
\hline Female & $185(48.6 \%)$ & $196(51.4 \%)$ & $381(100 \%)$ & 0.463 \\
\hline Male & $187(51.5 \%)$ & $176(48.5 \%)$ & $363(100 \%)$ & \\
\hline \multicolumn{5}{|l|}{ Tooth location [n (\%)] } \\
\hline Mandibular & $158(47.5 \%)$ & $175(52.5 \%)$ & $333(100 \%)$ & 0.238 \\
\hline Maxillary & $214(52.1 \%)$ & $197(47.9 \%)$ & $411(100 \%)$ & \\
\hline \multicolumn{5}{|c|}{ Previous surgical site infection [n (\%)] } \\
\hline Yes & $339(50 \%)$ & $339(50 \%)$ & $678(100 \%)$ & 0.999 \\
\hline No & $33(50 \%)$ & $33(50 \%)$ & $66(100 \%)$ & \\
\hline \multicolumn{5}{|l|}{ Smoking [n (\%)] } \\
\hline Smoker & $152(49.8 \%)$ & $153(50.2 \%)$ & $305(100 \%)$ & 0.999 \\
\hline Non-smoker & $220(50.1 \%)$ & $219(49.9 \%)$ & $439(100 \%)$ & \\
\hline \multicolumn{5}{|c|}{ Traumatic extraction [n (\%)] } \\
\hline Yes & $51(50 \%)$ & $51(50 \%)$ & $102(100 \%)$ & 0.999 \\
\hline No & $321(50 \%)$ & $321(50 \%)$ & $642(100 \%)$ & \\
\hline
\end{tabular}

See more details in Table 3.

No patient had hypersensitivity to chlorhexidine, dysgeusia or tooth pigmentation.

\section{Discussion}

We conducted a randomised, controlled trial to assess the effectiveness of postoperative chlorhexidine
$0.12 \%$ mouthwash to reduce the prevalence of postextraction $\mathrm{AO}$, and we contrasted it with a placebocontrolled group. We presented new findings in which chlorhexidine treatment reduces the incidence of $A O$ by $63 \%$ in high-risk patients, with strong statistical power.

Subgroup analysis showed no difference in the incidence of $\mathrm{AO}$ and location of the extracted tooth. Mandibular teeth developed the same rate of $\mathrm{AO}$ as that of the maxillary teeth, and anterior teeth showed 
a similar prevalence to posterior teeth. These findings are consistent with our previous observations ${ }^{8}$. Therefore, our results can be extrapolated to any patient who requires an extraction, regardless of the location of the tooth.

$\mathrm{AO}$ incidence in this study was quite similar to that which we reported previously: $4.97 \%$ versus $6.4 \%{ }^{8}$. We suspect that the incidence might have been higher in the placebo group on this occasion (incidence was $7.26 \%$ ), since the study sample consisted of patients with an increased risk of developing alveolar osteitis. However, we can explain this because the placebo effect can work as an effective treatment, especially in conditions associated with pain ${ }^{15,22}$.

Our results have some similarities with those reported in the literature with similar interventions. Ragno and Szutnik ${ }^{18}$ (1991) reported a statistically significant reduction of $50 \%$ in the prevalence of $\mathrm{AO}$ after the extraction of mandibular third molars [risk ratio (RR) 0.5 ; $95 \%$ confidence interval (CI), 0.27 to 0.93]. Larsen ${ }^{13}$ (1991) studied the AO preventive effect of chlorhexidine $0.12 \%$ mouthwash after mandibular third molar removal and observed a reduction in the prevalence of $60 \%$ (RR $0.4 ; 95 \% \mathrm{CI}, 0.21$ to 0.75 ). Hermesch, et al. ${ }^{10}$ (1998) reported that chlorhexidine $0.12 \%$ mouthwash reduced to $38 \%$ the prevalence

Table 2- Frequency of patients by matched risk factors for alveolar osteitis, distributed by treatment group

\begin{tabular}{cccc}
\hline Risk factor & $\begin{array}{c}\text { Chlorhexidine } \\
(\mathbf{n = 3 7 2})\end{array}$ & $\begin{array}{c}\text { Placebo } \\
(\mathbf{n}=\mathbf{3 7 2})\end{array}$ & $\begin{array}{c}\text { Total } \\
(\mathbf{n}=\mathbf{7 4 4})\end{array}$ \\
\hline S & 24 & 24 & 48 \\
PI & 191 & 190 & 381 \\
TE & 3 & 3 & 6 \\
S+PI & 106 & 107 & 213 \\
\hline S+TE & 6 & 6 & 12 \\
\hline PI+TE & 26 & 26 & 52 \\
\hline S+PI+TE & 16 & 16 & 32 \\
\hline Total & 372 & 372 & 744 \\
\hline
\end{tabular}

S: Tobacco smoke

PI: Previous infection in surgical site

TE: Traumatic extraction of $\mathrm{AO}$ after extraction of impacted mandibular third molars (RR $0.62 ; 95 \% \mathrm{CI}, 0.40$ to 0.96 ). In contrast, Delilbasi, et al. ${ }^{7}$ (2002) found that $0.2 \%$ chlorhexidine had no statistically significant effect in reducing the prevalence of $\mathrm{AO}$ after mandibular third molar removal (RR $0.88 ; 95 \% \mathrm{CI}, 0.45$ to 1.72 ). However, these studies have a high risk of bias ${ }^{6,9,24}$.

Our study has several strengths compared to those reported in the literature. To minimize selection bias, we randomly included only the patients who had the risk factors that we reported previously ${ }^{8}$. Thus, the preventive effect was studied in a group known to have a high risk of developing $\mathrm{AO}$, and not in persons who have not had chances to develop $A O$, regardless of receiving treatment or not.

We assigned participants to each group randomly, blindly and matched by risk factors. Thus, both groups had similar distribution in the risk of developing $A O$, and the results cannot be taken by a simple imbalance in the risk to develop $A O$ in any of the groups.

Additionally, we did not observe losses regarding follow-up or treatment withdrawals, minimising attrition bias risk. This was because we designed a protocol of four phone calls encouraging the patient to attend the clinical control, and our local community clinics allow to "retain" patients.

Studies that we compared previously used a model of impacted mandibular third molars. We consider that this model does not differ from ours, in which we included the extraction of any tooth in the mouth. The only difference is that in the extraction of impacted third molars, the risk factor "traumatic extraction" is exacerbated, which explains why the prevalence of AO in these studies is higher. In addition, we controlled the tooth location, which gives us the advantage that our results can be extrapolated to a wider population, and not be limited to only those procedures with a high level of tissue damage (i.e. mandibular third molar extraction).

Nonetheless, this study has some weaknesses. Firstly, it is difficult to ensure that patients have

Table 3- Incidence of alveolar osteitis in patients treated with chlorhexidine $0.12 \%$ mouthwash or placebo

\begin{tabular}{cccccc}
\hline & AO & Health & Total & ARR (95\% CI) & $\begin{array}{c}\text { NNT } \\
(\mathbf{9 5 \%} \text { CI) }\end{array}$ \\
\hline Chlorhexidine & 10 & 362 & 372 & $4.57 \%$ & 21.88 \\
Placebo & 27 & 345 & 372 & $(1.5-7.7)$ & $(13.0-69.3)$ \\
\hline Total & 37 & 437 & 744 & & $0.006^{*}$ \\
\hline
\end{tabular}

AO: Alveolar Osteitis; ARR: Absolute Risk Reduction; NNT: Number Needed to Treat; Cl: Confidence interval; *: p<0.05; power=0.822 (Fisher's exact test) 
followed the full treatment, because they administered themselves in their homes. To reduce the risk of poor treatment procedure, we phoned the patients once a day for the first $4 \mathrm{~d}$ to remind them to use the rinse. Additionally, we required them to bring the bottles of mouthwash to the dental control, to ensure that it was fully used. If this had not been the case, the patient would have been discarded from the follow-up. We used sterile water as placebo, which could have been a risk if the patient recognized it as such, but in the reinforcement phone calls, we did not detect this situation in any patient. Secondly, the operational definition of smokers was changed in relation to our previous study ${ }^{8}$ (i.e. consumption of $\geq 5$ cigarettes $24 \mathrm{~h}$ after extraction, to consumption of $\geq 5$ cigarettes $24 \mathrm{~h}$ before extraction). To include the patient as a smoker we had to know if they smoked previously.

It has been proposed that intra-alveolar application of chlorhexidine $0.2 \%$ gel may be an effective treatment to prevent $A \mathrm{O}^{11,20,21}$. However, the evidence supporting this preventive therapy has the same inconsistencies and weaknesses as the studies of chlorhexidine $0.12 \%$ mouthwash ${ }^{6}$. There is no biological plausibility that this form of treatment can be more effective, because a concentration of $0.2 \%$ shows no additional antibacterial benefits than the concentration of $0.12 \%{ }^{3}$, and the substantivity of the chlorhexidine mouthwash is sufficient to maintain its effect for $12 \mathrm{~h}^{4}$. There is no evidence that chlorhexidine may have a negative effect on haemostasis, and the mode of use as a mouthwash does not interfere with clot formation, since our application protocol begins 24 $\mathrm{h}$ after the extraction, when the clot has already been stabilized and begins to be replaced with granulation tissue $^{1}$.

With the strengths and weaknesses of our study, we conclude that the use of chlorhexidine $0.12 \%$ mouthwash after tooth extraction is highly effective compared with placebo mouthwash in preventing $\mathrm{AO}$ in high-risk patients. Clinical trials evaluating preventive effects should consider the risk factors in the study design to minimise risk of bias.

\section{Acknowledgments}

This clinical trial was self-funded by the authors. Austral University of Chile supported the fees of registration and publication.

\section{References}

1-Amler $\mathrm{MH}$. The time sequence of tissue regeneration in human extraction wounds. Oral Surg Oral Med Oral Pathol. 1969;27(3):309-18. 2-Balbuena L, Stambaugh KI, Ramirez SG, Yeager C. Effects of topical oral antiseptic rinses on bacterial counts of saliva in healthy human subjects. Otolaryngol Head Neck Surg. 1998;118(5):625-9.

3-Berchier CE, Slot DE, Van der Weijden GA. The efficacy of $0.12 \%$ chlorhexidine mouthrinse compared with $0.2 \%$ on plaque accumulation and periodontal parameters: a systematic review. J Clin Periodontol. 2010;37(9):829-39.

4-Bonesvoll P. Oral pharmacology of chlorhexidine. J Clin Periodontol. 1977;4(5):49-65.

5-Caso A, Hung L-K, Beirne OR. Prevention of alveolar osteitis with chlorhexidine: a meta-analytic review. Oral Surg Oral Med Oral Pathol Oral Radiol Endod. 2005;99(2):155-9.

6-Daly B, Sharif MO, Newton T, Jones K, Worthington HV. Loca interventions for the management of alveolar osteitis (dry socket). Cochrane Database Syst Rev. 2012;12:CD006968.

7-Delilbasi C, Saracoglu U, Keskin A. Effects of $0.2 \%$ chlorhexidine gluconate and amoxicillin plus clavulanic acid on the prevention of alveolar osteitis following mandibular third molar extractions. Oral Surg Oral Med Oral Pathol Oral Radiol Endod. 2002;94(3):301-4.

8-Halabí D, Escobar J, Muñoz C, Uribe S. Logistic regression analysis of risk factors for the development of alveolar osteitis. J Oral Maxillofac Surg. 2012;70(5):1040-4.

9-Hedström L, Sjögren P. Effect estimates and methodological quality of randomized controlled trials about prevention of alveolar osteitis following tooth extraction: a systematic review. Oral Surg Oral Med Oral Pathol Oral Radiol Endod. 2007;103(1):8-15.

10-Hermesch CB, Hilton TJ, Biesbrock AR, Baker RA, Cain-Hamlin J, McClanahan SF, et al. Perioperative use of $0.12 \%$ chlorhexidine gluconate for the prevention of alveolar osteitis: efficacy and risk factor analysis. Oral Surg Oral Med Oral Pathol Oral Radiol Endod. 1998;85(4):381-7.

11-Hita-Iglesias P, Torres-Lagares D, Flores-Ruiz R, Magallanes-Abad N, Basallote-Gonzalez M, Gutierrez-Perez JL. Effectiveness of chlorhexidine gel versus chlorhexidine rinse in reducing alveolar osteitis in mandibular third molar surgery. J Oral Maxillofac Surg. 2008;66(3):441-5.

12-Jaafar N, Nor GM. The prevalence of post-extraction complications in an outpatient dental clinic in Kuala Lumpur Malaysia - a retrospective survey. Singapore Dent J. 2000;23(1):24-8.

13-Larsen PE. The effect of a chlorhexidine rinse on the incidence of alveolar osteitis following the surgical removal of impacted mandibular third molars. J Oral Maxillofac Surg. 1991;49(9):932-7.

14-Ministerio de Salud. Guía Clínica AUGE de Urgencia Odontológica Ambulatoria. 2011.

15-Moore PA, Crout RJ, Jackson DL, Schneider LG, Graves RW, Bakos L. Tramadol hydrochloride: analgesic efficacy compared with codeine, aspirin with codeine, and placebo after dental extraction. J Clin Pharmacol. 1998;38(6):554-60.

16-Noroozi AR, Philbert RF. Modern concepts in understanding and management of the "dry socket" syndrome: comprehensive review of the literature. Oral Surg Oral Med Oral Pathol Oral Radiol Endod. 2009;107(1):30-5.

17-Oginni FO. Dry socket: a prospective study of prevalent risk factors in a Nigerian population. J Oral Maxillofac Surg. 2008;66(11):2290-5. 18-Ragno JR Jr, Szkutnik AJ. Evaluation of $0.12 \%$ chlorhexidine rinse on the prevention of alveolar osteitis. Oral Surg Oral Med Oral Pathol. $1991 ; 72(5): 524-6$.

19-Shao J, Chow S-C, Wang H. Sample size calculations in clinical research. 2. ed. New York: Chapman \& Hall; 2003.

Authors declare no conflict of interest. 
20-Torres-Lagares D, Gutierrez-Perez JL, Infante-Cossio P, GarciaCalderon M, Romero-Ruiz MM, Serrera-Figallo MA. Randomized, double-blind study on effectiveness of intra-alveolar chlorhexidine gel in reducing the incidence of alveolar osteitis in mandibular third molar surgery. Int J Oral Maxillofac Surg. 2006;35(4):348-51.

21-Torres-Lagares D, Infante-Cossio P, Gutierrez-Perez JL, Romero-Ruiz MM, Garcia-Calderon M, Serrera-Figallo MA. Intra-alveolar chlorhexidine gel for the prevention of dry socket in mandibular third molar surgery. A pilot study. Med Oral Patol Oral Cir Bucal. 2006;11(2):E179-84.
22-Turner JA, Deyo RA, Loeser JD, Von Korff M, Fordyce WE. The importance of placebo effects in pain treatment and research. JAMA. 1994;271(20):1609-14.

23-Upadhyaya C, Humagain $\mathrm{H}$. Prevalence of dry socket following extraction of permanent teeth at Kathmandu University Teaching Hospital (KUTH), Dhulikhel, Kavre, Nepal: a study. Kathmandu Univ Med J (KUMJ). 2010;8(29):18-24.

24-Yengopal V, Mickenautsch S. Chlorhexidine for the prevention of alveolar osteitis. Int J Oral Maxillofac Surg. 2012;41(10):1253-64. 